Editorial

\title{
Premature Ovarian Insufficiency and Bone Health Care: A Concern of the Gynecologist
}

\section{Insuficiência ovariana prematura e os cuidados com a saúde óssea: uma preocupação do ginecologista}

\author{
Marcos Felipe Silva de Sá ${ }^{1}$ \\ 1 Universidade de São Paulo, Ribeirão Preto, SP, Brazil \\ Rev Bras Ginecol Obstet 2018;40:305-308.
}

The greatest increase in bone mass occurs during puberty, and the amount of bone gained during adolescence is the major contribution to the peak bone mass (PBM) that occurs around the age of 30-35 years old. Studies conducted by the National Osteoporosis Foundation have shown the importance of the timing of the PBM, as it determines the phase of the life cycle in which the bone mass is optimized. ${ }^{1}$ In healthy girls, the earlier the onset of puberty, the greater the body mass and the bone mineral density (BMD) at the completion of skeletal maturity. ${ }^{2-5}$

The PBM varies according to the location in the skeleton. Estimates based on longitudinal studies performed by the Canadian Multicentre Osteoporosis Study showed the PBM for the lumbar region occurs between 33 and 40 years of age, and that the PBM for the hip occurs between 16 and 19 years of age. $^{6}$

PBM is influenced by genetic factors, nutritional status, adequate endocrine function, and physical activity, and is the major determinant of the future risk of fractures in elderly women. ${ }^{7}$ Among the endocrine factors are gonadal, adrenal and pituitary hormones; and, in women, estradiol plays a key role. Estradiol acts on the bones through several mechanisms and exerts an antiresorptive action. ${ }^{8}$ According to some authors, estrogens also act on the bones by indirect mechanisms through an action in the muscles by evidencing an interrelationship between mechanical forces and the action of steroids and growth factors on the tissue masses of both the bones and the muscles. ${ }^{9}$

Several clinical situations that lead to hypoestrogenism are associated with BMD loss by leading to osteopenia and osteoporosis. The most typical known situation is the menopausal period. However, when hypoestrogenism occurs in the pubertal period and in adolescence, it may result in a PBM reduction in these young women. Amenorrheic adolescents have a lower

Address for correspondence Marcos Felipe Silva de Sá, MD, $\mathrm{PhD}, \mathrm{Av}$. Bandeirantes, 3900, 14049-900, Monte Alegre,

Ribeirão Preto, SP, Brazil

(e-mail: marcosfelipe@fmrp.usp. br).
BMD compared to those who menstruate regularly. The earlier the hypoestrogenic condition is established and the longer it is extended, the greater the repercussions on bone mass, with an increased risk of fractures. Several conditions can lead to hypoestrogenism in young women, such as hypothalamic amenorrhea, hyperprolactinemia, and premature ovarian insufficiency (POI), among others. ${ }^{8,10,11}$

Premature ovarian insufficiency is a clinical syndrome defined by the depletion of the follicular activity before the age of 40 years old. It is characterized by amenorrhea, increased gonadotrophins (follicle-stimulating hormone [FSH] $>25 \mathrm{mIU} / \mathrm{mL}$ ) and low levels of estradiol. ${ }^{11}$ The incidence of $\mathrm{POI}$ in the general population is $1 \%$, and it represents $6 \%$ to $10 \%$ of the causes of amenorrhea in general, and $10 \%$ to $15 \%$ of the causes of primary amenorrhea. There is a family history of the disease in $4 \%$ of the patients. ${ }^{11}$ Patients with POI have a pattern in bone turnover markers similar to the one found in the menopausal state. ${ }^{12-18}$ This is an important concern for the health of young women with POI, particularly if they have not yet reached PBM.

Compared to women who experience menopause at normal ages, patients with POI have a 1.5 -fold greater risk of fracture. ${ }^{19}$ Some studies have shown a lower BMD in women with POI or in the menopause before the age of 45 years old by any etiology. Compared to women who menstruate regularly, women with POI, karyotype 46, XX (mean age: 32 years; range: 20-39 years) had significantly lower BMD Z-scores. About $20 \%$ of these women had a BMD Z-score $<2.0$, which indicates a low BMD for their age and a fracture risk factor. ${ }^{20}$

A delay in the diagnosis greatly contributes to worsening the BMD. ${ }^{21}$ It is very common to find patients with amenorrhea who have already lost precious time in doctors' offices and basic health units without the doctor investigating for a diagnosis of POF. In cases of amenorrhea, the possibility of 
POI should always be considered, and an effective search should be performed regarding the clinical picture (the climacteric symptoms of women) and high dosage of serum FSH. After the POI diagnosis, bone vitality should always be addressed because a loss in bone mass may have already occurred, and this should be a primary concern with the health of young women with POI. ${ }^{22-24}$

The treatment for osteopenia and osteoporosis caused by hypoestrogenism is essential and fundamentally based on the administration of estrogen replacement, which is indicated as a mandatory procedure as long as formal contraindications and patient acceptance are respected. ${ }^{11,25}$

Densitometry is directly related to estradiol levels. ${ }^{26}$ Bone mineral density correlates positively with body fat (\%), fat distribution and estradiol levels, and estradiol and age were among the factors associated with L2-L4 BMD. ${ }^{27}$ Levels below $20 \mathrm{pg} / \mathrm{mL}$ may have protective effects on the bone mass. Women with undetectable levels of estradiol $(<5 \mathrm{pg} / \mathrm{mL})$ were at a 2.5-fold higher risk of fracture compared to women with estradiol levels between $5 \mathrm{pg} / \mathrm{mL}$ and $25 \mathrm{pg} / \mathrm{mL}^{28,29}$

Thus, smaller estrogen dosages are required to meet bone maintenance needs. Low doses of estrogen, especially when associated with calcium, have a positive effect on bone mass, and its action appears to be predominantly on reabsorption, but not on bone formation after the age of 70 years old. ${ }^{30}$

We must always remember that there are several other goals of hormone replacement therapy (HRT) besides prevention and treatment of secondary bone loss due to ovarian failure. Therefore, the needs of each patient should be taken into account in order to define the estrogen dose to be administered. Further studies are needed to prove the efficacy of lower estrogen doses for cardiovascular protection, vasomotor phenomena, etc.

A three-year prospective randomized clinical trial was conducted by the United States National Institutes of Health (NIH) in young women with POI, karyotype $46, \mathrm{XX}$, in order to investigate the efficacy of a standardized HRT regime for BMD treatment. The study used transdermal E2 replacement $(100 \mu \mathrm{g} /$ day $)$ with cyclic oral progestogen $(10 \mathrm{mg}$ oral medroxyprogesterone acetate daily for 12 days per month). This replacement therapy improved the BMD of the lumbar spine and of the femoral neck, so that at the end of the threeyear intervention, the BMD did not differ between women with POI and the control group. ${ }^{31}$

The treatment of POI can have different doses and dosages according to the life period of onset of the disease. There is no evidence of which is the best route, oral or transdermal, and what is the best therapeutic regimen. ${ }^{11,32-35}$ In patients diagnosed in the pubertal period and without adequate development of secondary sexual characteristics, puberty should be induced with a low dose of $17 \beta$-estradiol and a gradual increase over a period of 2 to 3 years. Progestogens should be used two years after the onset of puberty induction with estradiol or as soon as the first menstrual bleeding occurs. In cases of later diagnosis, and with no remaining concern about growth, the initial estrogen dose may be higher and more rapidly progressive with increases every three to six months until the adult dose is reached. The recommendation is that the hormonal therapy simulates as close as possible the regular levels of ovarian estrogen production and its continuity until the natural menopause age. ${ }^{11,21}$

The use of combined oral contraceptives (COCs) is an alternative to the conventional treatment with natural estrogens. In cases of adolescents, who are still in the development phase of the PBM, some studies have shown that COCs may have a less positive impact on the BMD. ${ }^{36}$ However, further studies are needed to prove this effect..

A point to consider is the inclusion or not of BMD in the propaedeutic routine of patients with POI, especially those affected by the disease during adolescence and/or those with additional risk factors. Although BMD measurement is the gold standard for bone mass evaluation, and despite the large number of publications clearly pointing to bone loss, there is no consensus regarding the need to routinely indicate BMD measurement in the evaluation and follow-up of patients with POI.

According to Cox and Liu, ${ }^{35}$ "as a consequence of decreased estrogen levels, women with POI often do not achieve peak bone density and may experience loss of bone mass. If hormone therapy is initiated and the woman has not experienced fractures, it is not necessary to do bone mineral density testing."

On the other hand, other authors indicate BMD examination after the diagnosis of POI. ${ }^{37,38}$ Torrealday et $\mathrm{al}^{39}$ suggest that BMD measurement may be useful and should be considered for women with POI already at the beginning of the approach. It should be repeated in those who decide to continue hormone therapy until the equivalent time of menopause for that population. In turn, the European Society for Human Reproduction and Embryology (ESHRE) ${ }^{11}$ recommends the initial BMD measurement. If the results are normal and the patient undergoes hormonal therapy immediately upon diagnosis, there is no need to repeat the measurement. If the BMD measurement indicates osteoporosis, once the HRT is initiated, the BMD measurement should be repeated after five years. If the BMD continues to decline even with estrogen therapy, the conduct should be reviewed, and other factors that trigger osteoporosis should be sought.

The cost-benefit of measuring BMD in osteoporosis screening to assess its benefit as a prevention method for fractures in women is questioned. Most cohort studies to assess the use of BMD for this purpose included patients older than 65 years of age. ${ }^{40}$ For these patients, by considering the cut-off point of 2 standard deviations, the sensitivity is $9 \%$, the specificity is $99 \%$, and the positive predictive value is $56 \%$. Therefore, the BMD can predict the risk of fracture, but has low accuracy to identify individuals who will (or will not) have fractures. ${ }^{41}$

However, there are currently no alternatives to BMD for this evaluation, since bone turnover markers do not have well-established reference standards yet, given the variations observed among the various studies. ${ }^{12-18}$

In the Brazilian Unified Health System (SUS, in the Portuguese acronym), BMD measurement is authorized in some special situations, ${ }^{42}$ including cases of hypogonadism in men and women, postmenopausal women with risk factors, and 
to monitor changes in bone mass due to the course of osteoporosis and the different treatments available for this disease. Therefore, there is a possibility of access to the measurement of BMD, even if using public services, but also practical difficulties to perform the exam because it has a high cost and, in Brazil, few public services are available to the population.

Many questions remain unanswered given the lack of scientific evidence:

Are there differences in the behavior of bone mass over time when comparing women with POI and those who experienced menopause at the natural time?

Can we extrapolate to women with POI the sensitivity, specificity and predictive values for predicting fractures obtained with the BMD measurement performed in postmenopausal women?

Is it justified to perform a BMD measurement in young women with POI?

The evidence of the association of hypoestrogenism and low bone density and its association with the increased risk of fractures could be a justification for dispensing patients from undergoing a BMD measurement before starting hormone therapy?

It is known that estrogen therapy may fail in some patients, since other factors may interfere with the maintenance or loss of bone mass. How can we be sure that the patient undergoing hormone therapy will be protected from bone loss if she is not monitored through BMD measurements?

Are there alternative ways to confirm that patients with POI are already losing bone mass without BMD measurements?

\section{Conclusion}

In the literature, there are no evidence-based guidelines on criteria to maintain bone health in women with POI. It has not been definitively demonstrated that a reduced BMD in POI is indicative of an association of the disease with an increased fracture risk because the evidence is based on short-term observations and expert opinion. In fact, studies with the clear aim to clarify this cause-effect relationship are difficult to perform because they would involve ethical issues (for example, failure to treat patients on estrogen therapy as a control group), or the high cost and long duration of the follow-up, since the patients should be observed for long periods.

Moreover, the results of BMD studies performed in postmenopausal women cannot be extrapolated to a population of young women with estrogen deficiency before the age of 40 years old in order to predict fractures that will occur 20 to 30 years later, when other risk factors for fractures may be involved.

Despite the lack of such evidence with long-term randomized clinical trials, common sense suggests that the physician should rely on existing data in the literature, especially the guidelines of specialty societies.

The review of the literature shows that the consulted studies are practically consensual about these aspects of the POI approach. Estrogen replacement therapy should begin immediately after diagnosis, obviously respecting the con- traindications to its use. The BMD measurement for an initial evaluation before starting hormone therapy would be a good practice. However, if the patient's access to this test is difficult, she can be dispensed by considering the unquestionable benefits of estrogens on bone mass, even in very small doses. The risks of treatment failure should be carefully ascertained in view of the possibility of associated comorbidities or other factors interfering with bone mass.

More than half of the women with POI have inadequate vitamin D levels and low calcium intake. Many are not adherent to hormone therapy, do not exercise regularly, and may be smokers. Therefore, to ensure good bone mass, in addition to hormone therapy, women with POI should maintain a healthy lifestyle that involves physical exercise, abstinence from smoking, a balanced diet with good intake of foods rich in calcium and vitamin D, and weight control.

\section{References}

1 Weaver CM, Gordon CM, Janz KF, et al. The National Osteoporosis Foundation's position statement on peak bone mass development and lifestyle factors: a systematic review and implementation recommendations. Osteoporos Int 2016;27(04):1281-1386. Doi: 10.1007/s00198-015-3440-3

2 Gilsanz V, Chalfant J, Kalkwarf H, et al. Age at onset of puberty predicts bone mass in young adulthood. J Pediatr 2011;158(01): 100-105, 105.e1-105.e2. Doi: 10.1016/j.jpeds.2010.06.054

3 Gilsanz V, Gibbens DT, Carlson M, Boechat MI, Cann CE, Schulz EE. Peak trabecular vertebral density: a comparison of adolescent and adult females. Calcif Tissue Int 1988;43(04):260-262. Doi: 10.1007/BF02555144

4 Matkovic V, Jelic T, Wardlaw GM, et al. Timing of peak bone mass in Caucasian females and its implication for the prevention of osteoporosis. Inference from a cross-sectional model. J Clin Invest 1994;93(02):799-808. Doi: 10.1172/JCI117034

5 Sabatier JP, Guaydier-Souquières G, Laroche $D$, et al. Bone mineral acquisition during adolescence and early adulthood: a study in 574 healthy females 10-24 years of age. Osteoporos Int 1996;6 (02):141-148

6 Berger C, Goltzman D, Langsetmo L, et al; CaMos Research Group. Peak bone mass from longitudinal data: implications for the prevalence, pathophysiology, and diagnosis of osteoporosis. J Bone Miner Res 2010;25(09):1948-1957. Doi: 10.1002/jbmr.95

7 Brandão CMA, Vieira JGH. Fatores envolvidos no pico de massa óssea. Arq Bras Endocrinol Metabol 1999;43:401-408. Doi: 10.1590/S0004-27301999000600003

8 Meczekalski B, Podfigurna-Stopa A, Genazzani AR. Hypoestrogenism in young women and its influence on bone mass density. Gynecol Endocrinol 2010;26(09):652-657. Doi: 10.3109/095135 90.2010 .486452

9 Carson JA, Manolagas SC. Effects of sex steroids on bones and muscles: Similarities, parallels, and putative interactions in health and disease. Bone 2015;80:67-78. Doi: 10.1016/j.bone.2015.04.015

10 Hergenroeder AC. Bone mineralization, hypothalamic amenorrhea, and sex steroid therapy in female adolescents and young adults. J Pediatr 1995;126(5 Pt 1):683-689. Doi: 10.1016/S00223476(95)70393-4

11 Webber L, Davies M, Anderson R, et al; European Society for Human Reproduction and Embryology (ESHRE) Guideline Group on POI. ESHRE Guideline: management of women with premature ovarian insufficiency. Hum Reprod 2016;31(05):926-937. Doi: 10.1093/humrep/dew027

12 Naylor K, Eastell R. Bone turnover markers: use in osteoporosis. Nat Rev Rheumatol 2012;8(07):379-389. Doi: 10.1038/nrrheum.2012.86 
13 Kurtoglu-Aksoy N, Akhan SE, Bastu E, et al. Implications of premature ovarian failure on bone turnover markers and bone mineral density. Clin Exp Obstet Gynecol 2014;41(02):149-153

14 Eastell R, Garnero P, Audebert C, Cahall DL. Reference intervals of bone turnover markers in healthy premenopausal women: results from a cross-sectional European study. Bone 2012;50 (05):1141-1147. Doi: 10.1016/j.bone.2012.02.003

15 Li M, Li Y, Deng W, et al. Chinese bone turnover marker study: reference ranges for C-terminal telopeptide of type I collagen and procollagen I N-terminal peptide by age and gender. PLoS One 2014;9(08):e103841. Doi: 10.1371/journal.pone.0103841

16 de Papp AE, Bone HG, Caulfield MP, et al. A cross-sectional study of bone turnover markers in healthy premenopausal women. Bone 2007;40(05):1222-1230. Doi: 10.1016/j.bone.2007.01.008

17 Glover SJ, Garnero P, Naylor K, Rogers A, Eastell R. Establishing a reference range for bone turnover markers in young, healthy women. Bone 2008;42(04):623-630. Doi: 10.1016/j.bone.2007.12.218

18 Glover SJ, Gall M, Schoenborn-Kellenberger O, et al. Establishing a reference interval for bone turnover markers in 637 healthy, young, premenopausal women from the United Kingdom, France, Belgium, and the United States. J Bone Miner Res 2009;24(03): 389-397. Doi: 10.1359/jbmr.080703

19 van Der Voort DJ, van Der Weijer PH, Barentsen R. Early menopause: increased fracture risk at older age. Osteoporos Int 2003; 14(06):525-530. Doi: 10.1007/s00198-003-1408-1

20 Popat VB, Calis KA, Vanderhoof VH, et al. Bone mineral density in estrogen-deficient young women. J Clin Endocrinol Metab 2009; 94(07):2277-2283. Doi: 10.1210/jc.2008-1878

21 Newson LR, Lewis R. Premature ovarian insufficiency: why is it not being diagnosed enough in primary care? Br J Gen Pract 2018; 68(667):83. Doi: $10.3399 /$ bjgp18 $\times 694661$

22 Anasti JN, Kalantaridou SN, Kimzey LM, Defensor RA, Nelson LM. Bone loss in young women with karyotypically normal spontaneous premature ovarian failure. Obstet Gynecol 1998;91(01): 12-15. Doi: 10.1016/S0029-7844(97)00552-8

23 Gallagher JC. Effect of early menopause on bone mineral density and fractures. Menopause 2007;14(3 Pt 2(:567-571. Doi: 10.10 97/gme.0b013e31804c793d

24 Ohta H, Sugimoto I, Masuda A, et al. Decreased bone mineral density associated with early menopause progresses for at least ten years: cross-sectional comparisons between early and normal menopausal women. Bone 1996;18(03):227-231

25 Sullivan SD, Sarrel PM, Nelson LM. Hormone replacement therapy in young women with primary ovarian insufficiency and early menopause. Fertil Steril 2016;106(07):1588-1599. Doi: 10.1016/ j.fertnstert.2016.09.046

26 Ettinger B, Pressman A, Sklarin P, Bauer DC, Cauley JA, Cummings SR. Associations between low levels of serum estradiol, bone density, and fractures among elderly women: the study of osteoporotic fractures. J Clin Endocrinol Metab 1998;83(07):2239-2243. Doi: 10.1210/jcem.83.7.4708

27 Luo X, Cheng R, Zhang J, et al. Evaluation of body composition in POF and its association with bone mineral density and sex steroid levels. Gynecol Endocrinol 2018;•••:1-4. Doi: 10.1080/09513590. 2018.1473359
28 Reginster JY, Sarlet N, Deroisy R, Albert A, Gaspard U, Franchimont P. Minimal levels of serum estradiol prevent postmenopausal bone loss. Calcif Tissue Int 1992;51(05):340-343. Doi: 10.1007/ BF00316876

29 Cummings SR, Nevitt MC, Browner WS, et al; Study of Osteoporotic Fractures Research Group. Risk factors for hip fracture in white women. N Engl J Med 1995;332(12):767-773. Doi: 10.1056/ NEJM199503233321202

30 Prestwood KM, Thompson DL, Kenny AM, Seibel MJ, Pilbeam CC, Raisz LG. Low dose estrogen and calcium have an additive effect on bone resorption in older women. J Clin Endocrinol Metab 1999;84(01):179-183. Doi: 10.1210/jcem.84.1.5416

31 Popat VB, Calis KA, Kalantaridou SN, et al. Bone mineral density in young women with primary ovarian insufficiency: results of a three-year randomized controlled trial of physiological transdermal estradiol and testosterone replacement. J Clin Endocrinol Metab 2014;99(09):3418-3426. Doi: 10.1210/jc.2013-4145

32 Steingold KA, Matt DW, DeZiegler D, Sealey JE, Fratkin M, Reznikov S. Comparison of transdermal to oral estradiol administration on hormonal and hepatic parameters in women with premature ovarian failure. J Clin Endocrinol Metab 1991;73(02):275-280. Doi: $10.1210 /$ jcem-73-2-275

33 Bondy CA; Turner Syndrome Study Group. Care of girls and women with Turner syndrome: a guideline of the Turner Syndrome Study Group. J Clin Endocrinol Metab 2007;92(01):10-25. Doi: $10.1210 / j c .2006-1374$

34 Panay N, Kalu E. Management of premature ovarian failure. Best Pract Res Clin Obstet Gynaecol 2009;23(01):129-140. Doi: 10.1016/j.bpobgyn.2008.10.008

35 Cox L, Liu JH. Primary ovarian insufficiency: an update. Int J Womens Health 2014;6:235-243. Doi: 10.2147/IJWH.S37636

36 Warholm L, Petersen KR, Ravn P. Combined oral contraceptives' influence on weight, body composition, height, and bone mineral density in girls younger than 18 years: a systematic review. Eur J Contracept Reprod Health Care 2012;17(04):245-253. Doi: 10.3 109/13625187.2012.692411

37 Rafique S, Sterling EW, Nelson LM. A new approach to primary ovarian insufficiency. Obstet Gynecol Clin North Am 2012;39(04): 567-586. Doi: 10.1016/j.ogc.2012.09.007

38 Nelson LM. Clinical practice. Primary ovarian insufficiency. N Engl J Med 2009;360(06):606-614. Doi: 10.1056/NEJMcp0808697

39 Torrealday S, Kodaman P, Pal L. Premature Ovarian Insufficiency an update on recent advances in understanding and management. F1000 Res 2017;6:2069. Doi: 10.12688/f1000research.11948.1

40 Marshall D, Johnell O, Wedel H. Meta-analysis of how well measures of bone mineral density predict occurrence of osteoporotic fractures. BMJ 1996;312(7041):1254-1259. Doi: 10.1136/ bmj.312.7041.1254

41 Silva LK. [Technology assessment in health care: bone densitometry and alternative therapies in post-menopausal osteoporosis]. Cad Saude Publica 2003;19(04):987-1003. Doi: 10.1590/ S0102-311 × 2003000400022

42 Ministério da Saúde. Portaria No 1.327, de 11 de novembro de 1999. http://bvsms.saude.gov.br/bvs/saudelegis/gm/1999/prt132 7_11_11_1999.html. Acessado dezembro 10, 2017 